\title{
Comparison of Gene Prediction Programs for Metagenomic Data
}

\author{
Non Yok,Gail Rosen \\ ECE Department, Drexel University \\ Philadelphia, PA, USA \\ ng39@drexel.edu,gailr@ece.drexel.edu
}

\begin{abstract}
This manuscript presents the most rigorous benchmarking of gene annotation algorithms for metagenomic datasets to date. We compare three different programs: GeneMark, MetaGeneAnnotator (MGA) and Orphelia. The comparisons are based on their performances over simulated fragments from hundred species of diverse lineages. We defined three different types of fragments: one type from the intra-coding region and the other types are from the gene edges. The general observation was that performances of all these programs improve as we increase the length of the fragment. On the other hand, intra-coding fragments of our data show a low annotation error in all of the programs if compared to the genes edges.
\end{abstract}

Keywords- Metagenomic; Orphelia; MGA; GeneMark; fragments, sensitivity; specificity; error

\section{INTRODUCTION}

Metagenomic analysis is defined as the characterization of microbial genomes via the direct isolation of genomic sequences from the environment without prior cultivation (1). There are three major software programs used in gene prediction in metagenomic: GeneMark $(3,4)$, MGA (6) and Orphelia(2). GeneMark heuristic uses 3-periodic zero order Markov model that works with codon frequencies table to predict genes in metagenomics (3). MGA is an upgrade version of another software package, called MetaGene(MG) (5)which is used in gene prediction in metagenomic sequence data. In MGA genes are predicted in two stages: First stage all ORFs are scored by their base compositions and lengths using the dicodon regression models (Bacteria, Archae, Phage, Self) (6). Second, an optimal (high-scored) combination of ORFs is calculated using additional statistics (6). However, Orphelia algorithm uses artificial neural network to predict genes in metagenomic reads (2). In this paper, we will benchmark these programs using artificially fragmented reads extracted from 100 species of diverse lineages. Hoff et al. used only 12 species in their comparison (2); therefore, their sample is too small to represent an environmental sample. Also, no predecessor has separately examined fragments that contain gene edges as opposed to intra-coding regions. Because our metagenomic data is diverse and fragmented to represent Sanger's reads and the next generation sequencing, the results of our experiments reveal some important issues that our predecessors did not discover.

\section{METHOD \\ A. Fragment Types}

We created artificial metagenomic fragments out of 100 organisms and grouped them according to their lengths: 100 to $700 \mathrm{bp}$ fragment groups. Next, we defined three different types of fragments of equal length from each group based on the order of coding and flanking regions in the fragment. We named them Type A, Type B and Type C. For instance, in 700 bp fragments, Type A fragment consists of flanking region of variable length, 300-400 bp followed by a coding region of a variable length $300-400 \mathrm{bp}$. The length of the flanking and the coding regions is determined randomly, but the length of the whole fragment must equal to $700 \mathrm{bp}$. Type B fragment is different from Type A, in that it consists purely of coding sequence and it is picked randomly from within a gene region.

\section{B. Performance Metrics}

The objective of this paper is to compare the performances of these software packages in gene prediction. The means of comparison are measures of sensitivity, specificity, and f-measure. In addition to two newly defined measures known as annotation and prediction errors.

$$
\text { annotationErr }=\frac{|L p-L g b|+|R p-R g b|}{|F g b|}
$$

Where Lp stands for the left end index of the gene annotation of the program, while Lgb stands for the left end index of the GenBank annotation. Rp stands for the right end index of the fragment annotation by the program, but Rgb stands for the right end index of the fragment annotation according to the GenBank. Fgb denotes fragment length according to the GenBank annotation.

predictionErr $=\frac{G m}{G t}$

Where Gm is the number of the missed genes by the program and $\mathrm{Gt}$ is the total number of genes

\section{RESULTS}


Tables 1 to 6 along with graph of f-measure and are the major results of the paper. These results reflect the performances of the software tools utilized in the experiment. Each table contains five different measured values with exceptions of Type B fragments, their tables contain only three measures and the $100 \mathrm{bp}$ fragments their tables does not contain annotation error. The measure is found insignificant due to the shortness of the fragments.

\begin{tabular}{|l|c|c|c|}
\hline \multicolumn{1}{|c|}{ measure } & GeneMark & MGA & Orphelia \\
\hline Annotation Err & 33.38 & 36.31 & 35.78 \\
\hline Sensitivity & 86.36 & 98.91 & 100 \\
\hline Specificity & 68.468 & 65 & 79.66 \\
\hline Prediction Err & 12 & 1 & 0 \\
\hline F-measure & 76.38 & 78.44 & 88.67 \\
\hline
\end{tabular}

Table 1: Performances of the three programs:GenMark, Orphelia and MGA over Type A fragments of 700 bp lengths. On this table Orphelia misses no genes; therefore, its sensitivity is $100 \%$

\begin{tabular}{|l|c|c|c|}
\hline \multicolumn{1}{|c|}{ measure } & GeneMark & MGA & Orphelia \\
\hline Annotation Err & 17.06 & 9.41 & 7 \\
\hline Sensitivity & 87.77 & 98.9 & 98.9 \\
\hline Prediction Err & 11 & 1 & 1 \\
\hline
\end{tabular}

Table 2: Performances of the three programs:GenMark, Orphelia and MGA over Type B fragments of 700 bp lengths. On this table, the prediction error of both Orphelia and MGA is only $1 \%$

\begin{tabular}{|l|c|c|c|}
\hline measure & GeneMark & MGA & Orphelia \\
\hline Sensitivity & 60.86 & 87.9 & 90.47 \\
\hline Spec ificity & 62.22 & 60.6 & 59.37 \\
\hline Prediction Err & 36 & 11 & 8 \\
\hline F-measure & 61.53 & 71.7 & 71.69 \\
\hline
\end{tabular}

Table 3: Performances of the three programs: Orphelia, MGA and GeneMark over fragments of Type $\mathrm{C}$ of lengths $700 \mathrm{bp}$. The sensitivity of Orphelia as well as MGA is $100 \%$

\begin{tabular}{|l|c|c|c|}
\hline measure & GeneMark & MGA & Orphelia \\
\hline Annotation Err & 23.37 & 13.6 & 22.83 \\
\hline Sensitivity & 88.29 & 100 & 100 \\
\hline Spec ificity & 83 & 85.8 & 88.18 \\
\hline Prediction Err & 11 & 0 & 0 \\
\hline F-measure & 85.56 & 92.3 & 93.71 \\
\hline
\end{tabular}

Table 4: Table 4: Performances of the three programs: Orphelia, MGA and GeneMark over fragments of Type A.

\begin{tabular}{|l|l|l|l|}
\hline \multicolumn{1}{|c|}{ measure } & GeneMark & MGA & Orphelia \\
\hline Sensitivity & 17.71 & 32.1 & 48.48 \\
\hline Specificity & 40.90 & 64 & 44.44 \\
\hline Prediction Err & 79 & 57 & 39 \\
\hline F-measure & 26.56 & 36 & 46.15 \\
\hline
\end{tabular}

Orphelia's sensitivity is the highest.
Table 5: Performances of the three programs: GeneMark, Orphelia and MGA over Type B fragments of $100 \mathrm{bp}$ lengths.

Table 6: Performances of the three programs: GeneMark,

\begin{tabular}{|l|c|c|c|}
\hline measure & GeneMark & MGA & Orphelia \\
\hline Sensitivity & 62.36 & 95.4 & 97.2 \\
\hline Prediction Err & 35 & 4 & 2 \\
\hline
\end{tabular}

Orphelia and MGA over Type B fragments of 100 bp lengths.

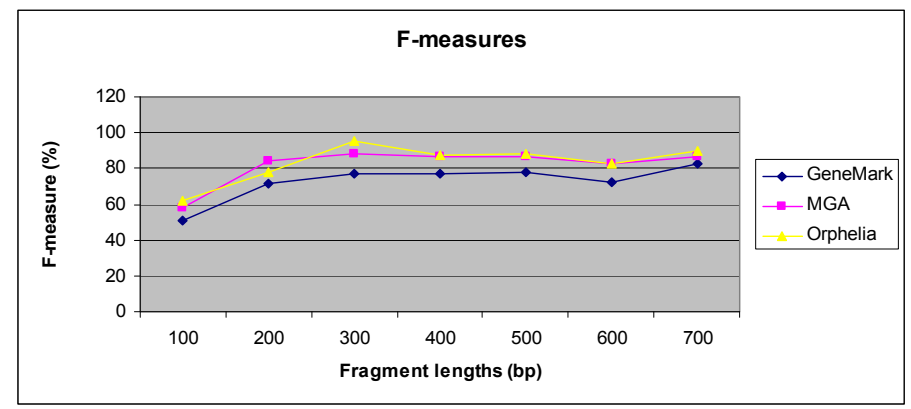

Figure 1: The graph of the f-measure graph of the three programs: Orphelia, MGA and GeneMark for fragments of lengths 100 to $700 \mathrm{bp}$.

\section{CONCLUSION}

The performances of GeneMark, MGA and Orphelia worsen with ultra short length fragments like those produced by Illumina Solexa. Overall, Orphelia performs the best for next-generation length reads.

\section{Acknowledgment}

This material is based upon work supported by the National Science Foundation under Grant No. \#0845827

\section{V1. REFERENCES}

[1] Thomas Lingner Rolf Daniel Burkhard Morgenstern Katharina J Hoff, Maike Tech and Peter Meinicke. Gene prediction in metagenomic fragments: A large scale machine learning approach. BioMed Central, page 14, 2008

[2] Peter Meinicke Katharina J. Hoff, Thomas Lingner and Maike Tech. Orphelia: predicting genes in etagenomic sequencing reads. Nucleic Acids Research, page 5, 2009.

I3] John Besemer and Mark Borodovsky. Heuristic approach to deriving models for gene finding. Nucleic Acids Res, page 10, 1999New York: Academic, 1963, pp. 271-350.

[4] Lukashin A. and Borodovsky M., GeneMark.hmm: new solutions for gene_finding, NAR, 1998, Vol. 26, No. 4, pp. 1107-1115.

[5] Jungho Park Hideki Noguchi and Toshihisa Takagi. Metagene: prokaryotic gene finding from environmental genome shotgun sequences. Nucleic Acids Res, Vol. 34,:No. 19 5623-5630, 2006.

6] Takeaki Taniguchi Hideki Noguchi and Takehiko Itoh. Metageneannotator: Detecting species-specific patterns of ribosomal binding site for precise gene prediction in anonymous prokaryotic and phage genomes. DNA RESEARCH, page 10, 2008. 